\title{
Future of Software Engineering
}

\author{
Pankaj Jalote \\ Director, Indraprastha Institute of Information Technology (IIIT), Delhi, \\ Professor, Department of Computer Science and Engineering \\ Indian Institute of Technology, Delhi, India
}

\begin{abstract}
Software and software business are undergoing a fundamental change, being driven by the limitations of current software models, ubiquity of computing, and increased penetration of wireless devices. At the same time, software has historically also shown a longevity and stickiness which other forms of technology have not shown. As software and software business evolve to respond to the new scenario, software engineering will have to change to deliver. Though any prediction is hard, and predicting in technology is even harder, this talk will look at how some aspects of software engineering might be in Future.
\end{abstract}

Bio: Pankaj Jalote is currently the Director of Indraprastha Institute of Information Technology (IIIT) Delhi, an autonomous Institute created through an act by Delhi Govt and with a State University status. Before joining IIIT, he was the Microsoft Chair Professor at Dept of Computer Science and Engineering at IIT Delhi. Earlier he was a Professor in the Department of Computer Science and Engineering at the Indian Institute of Technology Kanpur, India, where he was also the Head of the CSE Department from 1998 to 2002. From 85-89, he was an Assistant Professor at University of Maryland at college Park. From 1996 to 1998, he was Vice President at Infosys Technologies Ltd., a large Bangalore-based software house, and from 2003 to 2004, was a Visiting Researcher at Microsoft Corporation, Redmond, USA. He has a B.Tech. from IIT Kanpur, MS from Pennsylvania State University, and Ph.D. from University of Illinois at UrbanaChampaign. He is the author of four books including the highly acclaimed CMM in Practice, which has been translated in Chinese, Japanese, Korean etc, and the best selling textbook An Integrated Approach to Software Engineering. He is an advisor to many companies and is on the Technical Advisor Board of Microsoft Research India (Bangalore). His main area of interest is Software Engineering. He was on the editorial board of IEEE Transactions on Software Engineering and is currently on editorial board of Intl. Journal on Empirical Software Engineering and IEEE Trans. on Services Computing. He is a Fellow of the IEEE. 